\title{
DISCUSIÓN SOBRE LOS MODELOS DE ENSEÑANZA DE LA LECTURA EN LOS PRIMEROS GRADOS INSPIRADOS EN LA CONCIENCIA FONOLÓGICA
}

DISCUSSION ON THE MODELS OF EDUCATION OF THE READING IN THE FIRST DEGREES INSPIRED BY THE PHONOLOGICAL CONSCIENCE

Alfredo Altamirano ${ }^{1}$

Ministerio de Educación del Perú, Unidad de Medición de la Calidad Educativa

(RECIBIDo el 26/10/2010, ACEPTADo el 2/12/2010)

\begin{abstract}
RESUMEN
Este artículo pone en discusión la pertinencia para los primeros grados de la escolaridad de los modelos de enseñanza de la lectura surgidos a partir de las investigaciones sobre conciencia fonológica. Diversos estudios han planteado la existencia de distintos niveles implicados en la lectura. No obstante, estos niveles no son atendidos en los primeros grados desde la práctica pedagógica orientada por los modelos que aquí se discuten. Más bien, estos modelos apuntan a un aprendizaje casi exclusivo del plano léxico del lenguaje, dejando de lado los procesos de construcción semántica que tienen lugar cuando se lee. La conciencia fonológica es, en efecto, de vital importancia para la adquisición de lo escrito; sin embargo, para comprender lo que se lee no solo se requiere comprender las palabras del texto leído, sino, principalmente, construir el sentido del texto a medida que se leen las oraciones y los párrafos. A partir de la línea de trabajo que iniciara Emilia Ferreiro, se afirma que los niños de los primeros grados sí están en capacidad de llevar a cabo estos procesos de mayor complejidad, y la escuela debería promoverlos. De otro lado, se pone también en discusión la pertinencia didáctica de estos modelos, dado que es muy probable que se esté incidiendo en un aprendizaje repetitivo y poco significativo de la lectoescritura. Eso lleva a plantear la necesidad de reflexionar adecuadamente las relaciones entre investigación psicológica y práctica docente respecto de la lectoescritura. La pedagogía debería aprovechar los aportes psicológicos, pero debe complementarlos considerando otros criterios necesarios para propiciar el aprendizaje.
\end{abstract}

Palabras clave: Aprendizaje, lectura, conciencia fonológica, construcción semántica, pertinencia didáctica.

\begin{abstract}
This article raises questions about the relevance for the early grades of schooling models of reading instruction emerged from research on phonological awareness. Several studies have suggested the existence of different levels involved in reading. However, these levels are not seen in the early grades teaching practice guided by the models discussed here. Rather, these models point to an almost exclusive lexical learning of language, leaving aside the semantic construction processes that take place when reading. Phonological awareness is indeed
\end{abstract}

1 Especialista de evaluación en el área de Comunicación. E-mail: altamiran084_2@hotmail.com 
vital for the acquisition of writing, however, to understand what is read not only requires understanding the words of the text read, but mainly to build the meaning of the text as read the sentences and paragraphs. From the line of work that began Emilia Ferreiro, the article states that children in the early grades themselves are able to perform these more complex processes, and the school should promote them. On the other hand, it is also discussing the educational relevance of these models, since it is very likely to be affecting a very significant rote learning and literacy instruction. That has raised the need to reflect properly on the relationship between psychological research and teaching practices for literacy. The pedagogy should make use of psychological contributions, but it must complement them considering other criteria necessary to facilitate learning.

Keywords: Learning, reading, phonological anareness, semantic construction, relevance teaching.

\section{SITUACIÓN ACTUAL DE LA LECTURA EN LOS PRIMEROS GRADOS}

La lectura es una herramienta indispensable para el desarrollo de los individuos en el mundo actual. Por tal razón, la reflexión acerca de qué implica leer, cómo se llega a ser un buen lector y qué debe hacer el sistema educativo para desarrollar en todos los estudiantes una adecuada competencia lectora, cada vez es más relevante.

En ese sentido, hay muchos países que han mostrado grandes avances en lograr que todos sus estudiantes $-\mathrm{O}$, al menos, la gran mayoría- logren esta competencia. Otros países, en cambio, aún no han podido dar un salto cualitativo en esta materia. Un ejemplo de esto último es el caso del Perú. Para nadie es un misterio que la situación de la lectura en nuestro país es crítica. Nuestros estudiantes no logran un desarrollo adecuado de sus capacidades lectoras y no pueden utilizar estas herramientas para desempeñarse de manera óptima en su vida profesional, ciudadana e incluso personal. Diversas investigaciones (Gónzalez, 2006a; 2006b), así como evaluaciones de rendimiento nacionales e internacionales (MED, 2003; 2004; 2005a; 2005b) muestran que los logros en comprensión lectora obtenidos por los estudiantes peruanos que están a punto de culminar la educación secundaria son, por mucho, insatisfactorios.

Al observar lo que ocurre en el nivel primario, la situación es igual de dramática. La más reciente Evaluación Censal de Estudiantes 2009 realizada por el Ministerio de Educación (MED, 2010) muestra que, a nivel nacional, apenas un $23,1 \%$ de niños de segundo grado logra los aprendizajes mínimos esperados en comprensión lectora. Estos resultados son aún más alarmantes si se analizan únicamente los correspondientes a las escuelas públicas $(17,8 \%)$ y a las escuelas rurales $(11,6 \%)$. Incluso encontramos regiones donde el porcentaje de estudiantes que comprende lo que lee al nivel esperado no supera el $10 \%$.

Los resultados aquí señalados nos permiten comprender que el problema es estructural y atraviesa todos los grados de la educación primaria y secundaria. Podríamos afirmar entonces que la formación que reciben los alumnos una vez que ingresan a la escuela -y la que reciben en casa antes de ingresar a ella- no hace mucho por ayudarlos a leer más y mejor.

Toda esta realidad nos obliga a repensar los enfoques y programas pedagógicos que han de implementar los docentes con el fin de ayudar a sus estudiantes a desarrollar 
sus capacidades lectoras. En este artículo intentaremos hacer una revisión crítica de aquellos programas de enseñanza de la lectoescritura que parten de los hallazgos de las investigaciones sobre conciencia fonológica y que, de una forma u otra, conscientemente o no, influyen enormemente en la forma cómo la mayoría de docentes enseña a sus niños a leer.

\section{Los modelos de enseñanza de la lectura que parten de la conciencia fonológica}

Los modelos pedagógicos de conciencia fonológica plantean que la lectura implica cuatro tipos de procesos: perceptivos, léxicos, sintácticos y semánticos. Los procesos perceptivos nos permiten recibir e iniciar el procesamiento de los estímulos gráficos de lo escrito, los procesos léxicos nos permiten comprender las palabras percibidas, los procesos sintácticos permiten que comprendamos la estructura de las oraciones y a través de los procesos semánticos comprendemos el sentido de lo leído. Estos cuatro procesos actúan interrelacionándose y no necesariamente siguiendo una secuencia. Todos ellos son necesarios para la comprensión de lo leído (Cuetos, 1999).

Por lo tanto, para estos modelos, la enseñanza de la lectura debe apuntar al desarrollo de los cuatro procesos. En el caso de los niños que empiezan a acercarse a lo escrito, es de vital importancia la conciencia fonológica, al punto que esta sería el puente entre el lenguaje oral y la apropiación del lenguaje escrito. Entiéndase la conciencia fonológica como la conciencia que tiene el individuo de la composición fonémica del lenguaje oral: segmentación y secuencias fonémicas, aislamiento de los fonemas iniciales y finales, etc. (Bravo, 2003).

En consecuencia, estos programas apuntan a la necesidad de desarrollar la conciencia fonológica en los niños a los que les queremos enseñar a leer y a escribir. Este sería el paso previo a la adquisición de la escritura.

\section{Insuficiencia de los programas pedagógicos inspirados en la conciencia fonológica}

Es innegable que los modelos pedagógicos inspirados en la conciencia fonológica tienen una amplia base científica, sustentada con diversas investigaciones de fuerte base neurológica que explican algunos de los procesos cognitivos que ocurren al momento de leer. No hay lugar a discusión respecto de la activación de ciertas zonas cerebrales al momento de leer y del rol predictivo que tiene la conciencia fonológica en cuanto al aprendizaje formal de la escritura. Sin embargo, desde aquí defendemos una concepción mucho más amplia de la lectura, y es precisamente desde esa concepción que queremos señalar algunos aspectos $-\mathrm{y}$, principalmente, sus implicancias pedagógicas- que no son abordados por los modelos de conciencia fonológica. Creemos que estos modelos resultan insuficientes para dar cuenta del largo camino que supone la adquisición de la escritura por parte de los niños y para brindarles a los docentes un panorama suficientemente abarcador de todos los procesos comprometidos en este importante aprendizaje.

Probablemente, parte de esta insuficiencia se deba a una extrapolación directa y poco reflexiva de los hallazgos psicológicos al campo pedagógico. Quizá también tenga que ver la 
forma cómo definimos el objeto de estudio, a saber, el lenguaje escrito. Sea como fuere, pensamos que todos los valiosos aportes de las investigaciones psicológicas y de las neurociencias deberían ser analizados y repensados cada vez que se les quiera aprovechar en el terreno educativo, considerando una adecuada aplicación didáctica y el respeto de principios pedagógicos como el aprendizaje significativo, el rol de los conocimientos previos, etc.

\section{Concepciones sobre el objeto a enseñar: la lengua escrita}

Un primer cuestionamiento a los modelos pedagógicos que enfatizan la conciencia fonológica tiene que ver con la concepción de lenguaje escrito que manejan. Estos modelos plantean que, cuando leemos, ponemos en marcha procesos de tipo perceptivo, léxico, sintáctico y semántico. No obstante, cuando se aborda la lectura en los primeros grados, estos modelos ponen un marcado y hasta excluyente énfasis en los procesos perceptivos y léxicos, en desmedro de los procesos sintácticos y, especialmente, los semánticos ${ }^{2}$. Esto es de suma importancia porque precisamente un problema muy extendido -más aún en nuestro medio- es el del "analfabetismo funcional" (González, 2006a, 2006b), que compromete a todas aquellas personas que logran decodificar palabras pero que no comprenden el sentido global del texto que conforman, el cual es un plano que va mucho más allá que el de la palabra. Lamentablemente, si bien nadie estará en desacuerdo con que debemos lograr que los niños puedan comprender en toda su complejidad mensajes escritos apropiados para su nivel de desarrollo, lo cierto es que un modelo que enfatice el plano de las palabras y diga poco o nada sobre los planos textuales que están por encima de él (oraciones, párrafos, textos) trae muchas consecuencias en la práctica educativa. Por ejemplo, en nuestro país, muchos docentes consideran que a los niños primero se les debe enseñar únicamente a leer palabras, para recién luego pasar a enseñarles a comprender oraciones y textos ${ }^{3}$. Probablemente esto tenga relación con que los niños peruanos de segundo grado, casi en su totalidad, puedan comprender palabras y oraciones, pero, cuando se les plantean textos, empieza a haber porcentajes considerables de niños que no logran comprender ni siquiera los más sencillos (MED, 2003).

Algunos autores señalan que, en la medida que el niño domina cada vez más el reconocimiento de palabras, sus niveles de comprensión lectora aumentan, debido a que una mayor rapidez en el reconocimiento léxico permite contar con mayor espacio en la memoria a corto plazo para la ejecución de los procesos superiores (LaBerge y Samuels citados en Cuetos, 1999). Pareciera ser entonces que el concepto de lectura que tienen estos modelos considera las palabras como lo más importante en el lenguaje escrito.

No obstante, si bien las palabras son las unidades mínimas con sentido en un texto, es también cierto que un conjunto de palabras no hace una lengua, sino que esta supone una serie de elementos que estructuran esas palabras para formar mensajes con sentido. Pensar que las palabras son lo más importante del lenguaje escrito supone una peligrosa disección del lenguaje, una suerte de autopsia que hace perder de vista el importante rol

2 Por supuesto, esta exclusión no es declarada sino que más bien se refleja en los énfasis y en los vacíos que encontramos en los programas que aquí comentamos.

3 En el mejor de los casos, los docentes de los primeros grados intentan que los niños comprendan textos, aunque solo a niveles superficiales (literales) de comprensión. 
que cumple el resto de elementos del lenguaje escrito, y que también deben y pueden ser abordados por la enseñanza. De hecho, sería legítimo preguntarse si la relación entre el reconocimiento de palabras y la comprensión lectora se debe únicamente al factor léxico, o, quizá, tengamos que ese reconocimiento léxico, a su vez, se ve favorecido por los procesos sintácticos y semánticos que, definitivamente, tienen que estar presentes si decimos que se logró una adecuada comprensión lectora, en un proceso recursivo que no se aprecia en los modelos pedagógicos que aquí comentamos.

Son precisamente esos elementos estructuradores del lenguaje los que, volviendo al caso peruano, nuestros estudiantes no logran comprender cuando leen, y es por eso de suma importancia que los modelos de enseñanza que se propongan abarquen también estos otros aspectos que van más allá de la palabra. Si no, mantendremos las mismas prácticas pedagógicas cuyos resultados nos desalientan, prácticas pedagógicas totalmente opuestas a las que se realizan en países exitosos en cuanto a lograr que sus estudiantes comprendan lo que leen. Manning \& Manning (1991) describen interesantes prácticas pedagógicas realizadas por docentes de Canadá, Australia y Nueva Zelanda, países con altos niveles de comprensión lectora, según evaluaciones internacionales como PIRLS y PISA.

Se podría afirmar que lo expresado hasta este momento exagera ciertos vacíos en las teorías que inspiran los modelos de conciencia fonológica, y que da una imagen sesgada de lo que proponen. No obstante, la reducción del objeto de estudio no se limita a esto, sino que se da otra omisión relevante. Los modelos de conciencia fonológica entienden la lengua escrita como una transcripción, reproducción o codificación de la lengua oral. Bajo este concepto, la escritura no hace mucho más que recoger la oralidad y fijarla en un soporte tangible que la haga más duradera, menos volátil. Al respecto, Ferreiro \& Teberosky (2005) y Ferreiro (1991) han discutido desde hace muchos años esta concepción. Estas autoras señalan que, más que un simple código que transcribe la oralidad, la escritura es todo un sistema de representación. Al margen de si "código" nos representa o no una diferencia respecto de "sistema de representación", lo interesante es que esta última categoría evidenciaría la naturaleza especial de la escritura frente a otros códigos. Por ejemplo, la creación del código telegráfico supone una adaptación a otro formato de los elementos y relaciones de la escritura (lo mismo ocurre con otros "sistemas alternativos", como los códigos binarios para computadoras o los códigos secretos para usos militares). Estos sistemas no alteran nada sustancial del código que toman como base.

En cambio, existe otro tipo de códigos (para Ferreiro, "sistemas") que no se limitan a transcribir sino que constituyen una lógica propia, con sus características y peculiaridades, en un proceso que es además histórico y sociocultural. La escritura es precisamente uno de estos sistemas ${ }^{4}$ (Ferreiro, 1991). Este enfoque nos ofrece una mirada más completa de nuestro objeto de conocimiento: la lengua escrita. Para comprender mejor esto, presentaremos dos concepciones distintas frente a un mismo caso, de manera que se pueda evidenciar en qué medida la concepción de lengua escrita que defendemos sería más abarcadora y pertinente. Cuetos (1999) indica que "existen determinadas situaciones que demuestran lo realmente compleja que es la lectura. Una de ellas es cuando nos

4 Solo por dar algunos ejemplos, la coma vocativa, las mayúsculas y muchos otros usos ortográficos no tienen ningún correlato en la oralidad por lo que no pueden considerarse como transcripción de ella: son total invención (construcción) de la escritura y solo tienen funcionalidad en ella. 
acercamos a un idioma desconocido, sobre todo si tiene un sistema de escritura diferente del nuestro (ejemplo: árabe, chino, hebreo, etc.), ya que en muchos casos ni siquiera somos capaces de discriminar si son signos gráficos o simples ilustraciones. Si se trata de un sistema alfabético como el castellano (ejemplo, inglés o francés), al menos sabemos discernir dónde termina una palabra y comienza otra" (p. 15). De esta afirmación se concluiría que, para Cuetos y otros autores con la misma concepción del lenguaje escrito, el aprendizaje del lenguaje escrito comienza cuando podemos descifrar las letras, sílabas y palabras. No debería sorprendernos entonces que a los niños de los primeros grados se les enseñe prioritariamente a reconocer estos elementos de lo escrito. Ferreiro (1991), por su parte, plantea el mismo caso de lenguas que no cuentan con un sistema alfabético, pero dándole un enfoque distinto y, según nuestro entender, más completo. La autora señala que, si a un grupo de adultos les presentamos textos escritos en lenguas no alfabéticas, estos, pese a no comprender las palabras, sí podrían construir diversos significados relacionados con el soporte material en que aparecen estos mensajes escritos, la funcionalidad de diversos elementos gráficos, el posible propósito comunicativo del texto, etc., en un continuo proceso hipotético a partir de pistas textuales:

\begin{abstract}
First, they discuss the clasification of the object that they have in their hands: Is it a book (if so, what kind), a newspaper, a magazine, a pamphlet, or what? Once they have agreed on the classification, they begin to anticipate the organization of the content. If it is a newpaper, it must have sections (foreign and domestic affairs, sports, etc.); if it is a book, the title, the author's name, the printing press and the year of publication should appear at the beginning, and a table of contents at the back or the front. They invariably assume that the pages are renumbered, which permits them to find the graphic distinctions between numbers and letters. (...) And so it goes on (Ferreiro, 1991, p. 48).
\end{abstract}

Es decir, para esta autora, leer, acceder al mundo escrito es mucho más que decodificar y comprender mensajes expresados con letras sino que supone acceder a la lógica de toda una práctica cultural cuyos significados no se limitan a la convención de las letras impresas. Lo interesante es que estas aproximaciones son similares a las que realizan los niños cuando se acercan al mundo de lo escrito y a la lectura de un sistema que aún no comprenden, con la diferencia de que estos no cuentan con todo el saber de la práctica cultural de la escritura con que sí cuenta un adulto (Ferreiro, 1991). De todos modos, es importante reconocer que el niño no espera a llegar al colegio ni a haber desarrollado una serie de procesos cognitivos (por ejemplo, la conciencia fonológica) para empezar a construir significados, sobre todo lo que supone el lenguaje escrito, entendido de esta forma más compleja (Ferreiro \& Teberosky, 2005; Ferreiro, 1991).

Analizar y reconocer distintas concepciones de la lectura nos permitirá comprender mejor sus consecuencias pedagógicas. Si concebimos la escritura como un código que traduce la lengua oral, entonces se entiende el casi exclusivo (y excluyente) énfasis puesto en los primeros grados en lograr que los niños reconozcan la correspondencia de las letras con sus sonidos. $\mathrm{Si}$, en contraposición, concebimos la escritura como un sistema de representación y comprendemos su compleja lógica interna, pues entonces podremos comprender que el acercamiento a lo escrito realizado por los niños mucho antes de desarrollar sus habilidades para la decodificación (acercamiento que continúa luego 
de este aprendizaje específico) es similar incluso al realizado por la humanidad en el proceso histórico de construcción de este sistema, y tiene detrás una lógica, una razón de ser (Ferreiro, 1991). Esto, además, nos permitirá pensar modelos de enseñanza que ayuden a los niños a ir consolidando y superando las diversas etapas del largo proceso de adquisición de la escritura.

\section{Modelo conductista de enseñanza}

Pasemos ahora a revisar el tipo de enseñanza propuesta por los modelos pedagógicos inspirados en la conciencia fonológica. En principio, la discusión de cómo enseñarles a leer a los niños ha estado orientada principalmente a decidir qué método es el indicado: partir del reconocimiento previo de las letras (ruta fonológica) o de un reconocimiento global (ruta visual) (Ferreiro \& Teberosky, 2005; Bravo, 2003; Cuetos, 1999). La salida a este dilema resulta siendo, según algunos autores, la de incluir ambos métodos en un modelo interactivo que reconoce las dos rutas. Sin embargo, esta no es una real superación del problema, puesto que se mantiene el mismo enfoque general de enseñanza común a cualquiera de estos métodos, el cual resulta insuficiente para alcanzar un desarrollo de las habilidades lectoras en toda su complejidad (Ferreiro \& Teberosky, 2005).

Así, quienes propugnan este tipo de enseñanza terminan planteando -quizá sin proponérselo- una enseñanza conductista de la lectoescritura, basada en un esquema asociacionista. No es gratuito entonces que muchos programas de este tipo busquen "entrenar" a los niños para acercarse a lo escrito, a través de una repetición constante y muchas veces rutinaria de ejercicios orientados a que los niños puedan establecer la correspondencia grafem.fonema. El niño es expuesto continuamente a los estímulos gráficos propios de lo escrito hasta que luego, en algún momento, alcanza el logro esperado. No hay acción sobre los procesos mentales que permiten ese logro. Cuando se trata de desarrollar habilidades lectoras de tipo semántico ocurre algo similar: los docentes terminan enseñando técnicas o procedimientos rígidos de lectura (como si el acto de leer fuera el mismo siempre), en lugar de buscar el desarrollo de estrategias que les permitan a los estudiantes ser lectores eficaces en diversas situaciones de lectura posibles (Solé, 2004)5.

No obstante, un modelo de este tipo no puede dar cuenta de todo lo complejo que es el proceso de adquisición de la lectoescritura, ya que hay muchos aspectos del lenguaje escrito que el niño no puede aprender por simple imitación y asociación. Por ejemplo, es muy común escuchar que un niño diga "Se ha rompido" del mismo modo que dice "Se ha dormido". Por supuesto, el niño no comete el error de la primera frase por internalización de algo que escuchó en el exterior (errores de este tipo lo cometen niños

5 Solé establece una diferencia entre procedimiento y estrategia. El primero es una secuencia rígida y universal de pasos a seguir para lograr un objetivo. Por su parte, una estrategia es una secuencia variable de pasos, según la consideración de los objetivos a alcanzar y del contexto que rodean al problema. Una verdadera enseñanza de la lectura pasa por desarrollar la capacidad de aplicar estrategias y no de aprender procedimientos universales y aplicarlos de manera reflexiva e indiscriminada en cualquier caso. Por supuesto, la diferencia entre procedimientos y estrategias tiene que ver, más que con qué paso se sigue, con cómo se sigue, si esa de manera reflexiva, considerando cada problema específico. Así, el subrayar la idea principal de los párrafos que vamos leyendo podría ser una estrategia útil si esta aparece explícita, pero no si hay que construirla. Sin embargo, muchos lectores buscan siempre subrayar alguna frase por párrafo, asumiendo que siempre es posible hacerlo. Ellos estarían aplicando un procedimiento y no una estrategia. 
de diversos entornos socioculturales), ya que nadie dice "rompido". Más bien, lo que resulta claro es que el niño ha hecho un esfuerzo -que, dicho sea de paso, la escuela no ha tenido que pedírselo ni enseñárselo- por encontrar una regularidad en verbos similares. El caso de la regularización de los verbos es uno de los ejemplos más evidentes de que los niños no son meros receptores de saberes "correctos" sobre la lengua provenientes del exterior, sino que se aventuran a una construcción interna de dichos saberes. Por ende, el tipo de enseñanza que se necesita para desarrollar habilidades lingüísticas, y específicamente las lectoras debe ser una enseñanza fundamentalmente constructivista, que se proponga ayudar al niño en el proceso continuo de construcción de conocimientos sobre el lenguaje escrito.

Por supuesto, no pretendemos reforzar una estigmatización banal y maniquea del aprendizaje bajo el esquema conductista: simplemente queremos evaluar si este esquema es lo suficientemente potente como para explicar el aprendizaje de algo tan complejo como la lectura y la escritura. Nos queda claro que el entorno provee una serie de estímulos que pueden alentar u obstaculizar el aprendizaje de lo escrito; sin embargo, nos parece que este aprendizaje es fundamentalmente acción interna, y es pensando en ella que se debe orientar la práctica educativa. Como se señaló anteriormente, dominar lo escrito no pasa únicamente por la internalización de la asociación entre grafemas y fonemas, sino principalmente, por la construcción de un sentido tanto al escribir como al leer. Esta capacidad de construir sentidos requiere mucho más que una enseñanza que nos induzca a asociar letras y sonidos. Como es de común acuerdo a nivel teórico, esta construcción del sentido supone una serie de procesos cognitivos que sobrepasan aquellos que tienen que ver con la conciencia fonológica y la decodificación. Y estos otros procesos cognitivos no se desarrollan partiendo de un enfoque asociacionista (qué fácil sería revertir los problemas de comprensión lectora si así fuera). Poco de esto se refleja en los programas pedagógicos que enfatizan casi de manera excluyente el desarrollo de la conciencia fonológica en los niños.

\section{Reduccionismo del rol del alumno}

Complementando lo anterior, nos parece, además, que los modelos pedagógicos de desarrollo de la conciencia fonológica fallan en su severa reducción del rol del niño en su aprendizaje de lo escrito. Siguiendo a Ferreiro \& Teberosky (2005), Ferreiro (1991), Siegrist \& Sinclair (1991) y otros autores, pensamos que el niño trae consigo una serie de conocimientos sobre el lenguaje escrito desde antes de recibir instrucción formal. El niño construye hipótesis a partir de todo el sistema conceptual que ya posee sobre la escritura. Los modelos pedagógicos que cuestionamos aquí parecieran perder de vista el valor de los conocimientos previos de los niños para este aprendizaje, quizá en parte porque desconocen que estos existen también para el caso del lenguaje escrito.

Asimismo, la imagen del niño que nos ofrecen los modelos pedagógicos de conciencia fonológica es la de una tabula rasa que espera llegar a la escuela y recibir instrucción formal para recién entonces hacerse de ideas acerca del lenguaje escrito. Este no es un niño en el que veamos acción interna y consciente respecto de lo que recibe; es un niño que no construye nada ni antes ni después de aprender a leer. Como apuntan Ferreiro \& Teberosky (2005): 
Algo que hemos buscado en vano en esta literatura es el sujeto mismo: el sujeto cognoscente, el sujeto que busca adquirir conocimiento, el sujeto que la teoría de Piaget nos ha enseñado a descubrir. ¿Qué quiere decir esto? El sujeto que conocemos a través de la teoría de Piaget es un sujeto que trata activamente de comprender el mundo que lo rodea, y de resolver los interrogantes que este mundo le plantea. No es un sujeto que espera que alguien que posee un conocimiento se lo transmita, en un acto de benevolencia. Es un sujeto que aprende básicamente a través de sus propias acciones sobre los objetos del mundo, y que construye sus propias categorías de pensamiento al mismo tiempo que organiza su mundo (p. 28).

Por supuesto, todo esto tiene repercusiones pedagógicas. Así como los niños llevan a cabo un proceso de construcción de las nociones de número (Kamii, 1985), estos también realizan un proceso de construcción del lenguaje escrito. Cabe aclarar que, cuando hablamos de procesos de construcción, no estamos diciendo que el niño inventa lo que el lenguaje escrito es realmente, sino lo que el lenguaje escrito es para él. La enseñanza debería tomar en cuenta la complejidad de este proceso de construcción, para acompañar al niño en ese tránsito y darle ayudas pedagógicas que le permitan sacar más provecho del mismo.

\section{Vacíos en la descripción de las etapas de adquisición de lo escrito}

Líneas arriba mencionábamos que los modelos que estamos discutiendo no se aproximan ni aprovechan los conocimientos previos de los niños para el aprendizaje de la lengua escrita. Seguramente muchos se preguntarán qué saberes previos sobre la escritura puede tener un niño que, precisamente, aún no sabe leer ni escribir. Los conocimientos previos a los que nos referimos no tienen que ver únicamente con datos, información específica sobre un tema (dicho sea de paso, el niño que llega a la escuela ya tiene algunos de estos datos: sentido izquierd.derecha y arriba-abajo de la lectura, entre otros), sino que nos referimos a los conocimientos previos en un sentido mucho más amplio, que tiene que ver con todos los sistemas de relaciones que nos permiten organizar nuestros saberes e interpretar nueva información (Kamii, 1991).

El niño obtiene este conocimiento gracias a un largo camino progresivo de adquisición y familiarización con el lenguaje escrito. Los modelos que priorizan el desarrollo de la conciencia fonológica nos hablan de un "umbral lector" en el cual los niños cristalizan en la decodificación una serie de procesos cognitivos (conciencia fonológica, conciencia sintáctica, conciencia semántica, conocimiento de las letras, memoria verbal, conciencia alfabética), e inician el aprendizaje formal de la lectura (Bravo, 2003). Sin embargo, la idea de "umbral lector" solo nos ofrece una fotografía de momento de toda una larga carrera que comienza con logros básicos y fundamentales como, por ejemplo, la diferenciación entre dibujo y escritura. Ni siquiera el concepto de "umbral lector" evita cierta dicotomización del aprendizaje de lo escrito en dos momentos discontinuos: cuando no se sabe y cuando se sabe.

Cuetos (1999) plantea tres etapas en el proceso de adquisición de lo escrito. La primera es la etapa logográfica, en la que los niños identifican las palabras globalmente a partir de sus rasgos y el contexto donde se encuentran, y no a través de la identificación de las 
letras que la conforman. La segunda es la etapa alfabética, en la que los niños son capaces de segmentar las palabras en sus letras componentes y de asignar a cada letra el sonido que le corresponde. La última etapa es la ortográfica, en la que los niños identifican cada una de las letras que componen la palabra y, de hecho, detectan en seguida si se produce algún error en su orden.

En realidad, tal como nos muestran Ferreiro \& Teberosky (2005) y Ferreiro (1991), este proceso de adquisición es mucho más largo y complejo. Esta autora, continuando la labor de Piaget e incursionando en un terreno en el que este no investigó, ha establecido una serie de etapas por las que atraviesa el niño hasta que aprende a leer. Por ejemplo, esta autora nos da más aportes acerca de cómo el niño pasa de la etapa logográfica a la alfabética, qué retos enfrenta y cómo los resuelve ${ }^{6}$. En este caso específico, antes de poder asignar a las letras sus respectivos sonidos, el niño pasa primero por una etapa silábico alfabética, en la que le asigna una letra al sonido de una sílaba entera (en el caso de los niños hispanohablantes estudiados por Ferreiro, estos asignan vocales).

Las etapas planteadas por Ferreiro, así como la descripción de qué ocurre en el niño mientras transita entre estas etapas, han sido corroboradas por Kamii, Long, Manning \& Manning (1991) en niños angloparlantes, con resultados interesantes, ya que, además, de verificar la validez de las etapas de Ferreiro, nos brindan información adicional relevante para la adquisición de la escritura en el idioma inglés (por ejemplo, el hecho de que los niños angloparlantes, en la etapa silábico alfabética, en lugar de asignar vocales a los sonidos de las sílabas, les asignan consonantes, lo cual puede tener que ver con la naturaleza de esta lengua). Siegrist (citado en Siegrist \& Sinclair, 1991) y Siegrist \& Sinclair (1991) han hecho lo propio con niños franco parlantes. Gracias a este tipo de investigaciones se ha abierto un interesante campo de estudio en el que se busca escuchar al niño con mayor minuciosidad y comprender todo el proceso (activo, dinámico) que este sigue, no desde la óptica adulta de la corrección del saber sino desde la perspectiva de los problemas cognoscitivos que tiene que enfrentar el niño y de los modos cómo este acomoda o adecua sus esquemas para resolverlos. El reto consiste en plasmar todas estas interesantes investigaciones en modelos pedagógicos que potencien estas etapas. Al respecto, Teberosky (2001) y Tolchinsky \& Pipkin (2001) han realizado algunos trabajos en ese sentido. De todos modos, este es aún terreno fértil por explorar.

\section{CONCLUSIONES}

1. Hemos insistido en marcar la diferencia entre investigación psicológica y modelos pedagógicos. La Psicología hace mucho en aportar información acerca de qué ocurre cuando alguien aprende, mientras que es la Pedagogía la llamada a plantear los mejores modelos para que este aprendizaje se realice. Criticamos los modelos pedagógicos

6 Otro aporte importante de Ferreiro es el descubrimiento de la exigencia de los niños de las etapas iniciales de que haya por lo menos tres letras juntas para considerar tal conjunto como una palabra y como perteneciente al mundo de lo escrito. Eso permite discutir si, a nivel de enseñanza, sería pertinente usar palabras como "oso" o "sol", que estarían en el límite de aceptabilidad léxica de un niño, para enseñarles a leer, y ni qué decir con grupos de dos letras. A partir de esto último también habría que evaluar si es pertinente una enseñanza basada principalmente en el acercamiento sílabas aisladas, considerando que estas no tienen ningún sentido y que para muchos niños en estados iniciales ni siquiera son escritura (cuánto de estos niños están en aulas de primer grado de zonas muy deprimidas en nuestro país). 
inspirados en los hallazgos sobre conciencia fonológica, pero reconocemos la importancia de esta para la adquisición del lenguaje escrito.

2. Debemos someter a análisis y discusión todas las propuestas de programas de adquisición de lo escrito a un constante análisis que tome en cuenta el respeto de ciertos principios básicos que rigen todo aprendizaje, y que, por lo tanto, deberían regir toda enseñanza (aprendizaje significativo, activación de conocimientos previos, construcción del aprendizaje, acción interna de quien aprende). De esa forma, se potenciará mucho más cualquier aporte que tenga como intención elevar los bajos niveles lectores que, por ejemplo, tenemos en nuestro país. Esto abre también un espacio de discusión acerca de si la Pedagogía debe seguir asumiendo los aportes de la Psicología como si estos fueran en sí mismos soluciones pedagógicas o si, en realidad, debe darles "forma didáctica" para que repercutan en mejoras más significativas.

3. Resulta llamativo que haya un consenso unánime respecto sobre estos principios generales que deben cumplirse en el proceso de enseñanz.aprendizaje, al tiempo que estos mismos principios son dejados de lado en los tan difundidos modelos pedagógicos inspirados en la conciencia fonológica, que apuestan más bien por una enseñanza asociacionista poco significativa y poco pertinente. Por supuesto, esto no niega la importancia del desarrollo de la conciencia fonológica en los niños, sino que nos hace ver que el que un proceso mental esté probado no implica que el mejor modo de fomentarlo pase por un entrenamiento repetitivo del mismo.

4. En esa línea, nos parece que queda aún mucho por investigar acerca del impacto de diversos modelos de enseñanza. En el campo específico de la lectoescritura, nos parece que se ha hecho muy poco por plantear alternativas a modelos bastante tradicionales que, salvo algunas mejoras, no han cambiado en su esencia. Estudios comparativos en este terreno nos permitirían ver qué modelos pedagógicos son más pertinentes y despejar la duda de que los pocos modelos hasta ahora conocidos son simplemente mejores que la enseñanza no estructurada. Asimismo, estos modelos se verían enriquecidos con más investigación respecto del desarrollo de los procesos sintácticos y semánticos antes y durante la apropiación de lo escrito.

5. Quienes defienden los programas de enseñanza inspirados en la conciencia fonológica quizá afirmen que no niegan muchos de los aspectos aquí desarrollados. Sin embargo, creemos que el problema no radica en los postulados declarados sino en aquellos que se enfatizan en la práctica. Claramente, estos modelos no priorizan el desarrollo de procesos semánticos en los primeros grados, dejando la sensación de que este es un trabajo que corresponderá a grados futuros. Pensamos que en todos los grados, a toda edad, la enseñanza de la comprensión lectora (de la cual forma parte aquello que llamamos "lectoescritura") debería potenciar las diversas habilidades que esta realmente implica. Por ello, nos parece inconveniente mantener una suerte de modelo en escalera que establece una secuencia fija de prerrequisitos entre los aprendizajes.

6. La investigación sobre las distintas etapas de adquisición de lo escrito debe dar lugar a prácticas y modelos pedagógicos más adecuados. Esta discusión se vuelve más relevante si pensamos la educación en zonas rurales, en donde la asistencia al preescolar es escasa. Niños que por primera vez recibirían educación formal (la 
cobertura en educación inicial es aún baja) serían sometidos a una enseñanza que se apoyaría en un determinado desarrollo previo que estos niños no poseen. Los maestros, guiados por los enfoques tradicionales, no consideran en qué etapa de acceso a la escritura están los niños, sino que les dan un mismo tipo de instrucción a todos.

7. Finalmente, consideramos que los aportes de autores como Emilia Ferreiro, Hermina Sinclair, François Siegrist, entre otros, siguiendo el legado piagetano, han servido para ampliar nuestra mirada de lo que implica la lectura. Pese a que quienes defienden estas ideas han investigado muy poco los modelos de enseñanza de la lectoescritura, creemos que esta perspectiva permitiría asimilar mejor los distintos aportes que nos brindan las investigaciones psicológicas, entre ellas las que tratan sobre la conciencia fonológica, incluyéndolos en un enfoque mucho más completo.

\section{REFERENCIAS BIBLIOGRÁFICAS}

1. Bravo, L. (2003). Lectura inicial y psicología cognitiva. Santiago de Chile: Ediciones Universidad Católica de Chile.

2. Cuetos, F. (1999). Psicología de la lectura. Madrid: Editorial Escuela Española.

3. Ferreiro, E. \& Teberosky, A. (2005). Los sistemas de escritura en el desarrollo del niño. México, D.F.: Siglo XXI Editores.

4. Ferreiro, E. (1991). Literacy acquisition and the representation of language. En: C. Kamii, M. Manning \& G. Manning (eds.), Early literacy: a constructivist foundation for whole language. Washington: National Education Association of the United States.

5. González, R. (2006a). Analfabetismo funcional en estudiantes de Lima. En: Problemas psicolingüísticos en el Perú. Evidencias empíricas (Obras completas/ Volumen I). Lima: Norma Reátegui.

6. González, R. (2006b). Comprensión lectora en estudiantes universitarios iniciales. En: Problemas psicolingüísticos en el Perú. Evidencias empíricas (Obras completas/ Volumen I). Lima: Norma Reátegui.

7. Kamii, C. (1991). What is constructivism? En: C. Kamii, M. Manning \& G. Manning (Eds.), Early literacy: a constructivist foundation for whole language. Washington: National Education Association of the United States.

8. Kamii, C.; Long, R.; Manning, M. \& Manning, G. (1991). A constructivist analysis comparing Spanis.speaking and Englis.speaking children. En: C. Kamii, M. Manning \& G. Manning (Eds.), Early literacy: a constructivist foundation for whole language. Washington: National Education Association of the United States.

9. Kamii, C. (1985). Young children reinvent arithmetic. New York: Tachers College Press.

10. Manning, M. \& Manning, G. (1991). Reflections on the constructive process. En: C. Kamii, M. Manning \& G. Manning (eds.), Early literacy: a constructivist 
foundation for whole language. Washington: National Education Association of the United States.

11. Ministerio de Educación del Perú (2010). Resultados de la Evaluación Censal de Estudiantes 2009 - ECE 2009. Recuperado el 23 de noviembre de 2010 de http:// www2.minedu.gob.pe/umc/ece2009/Resultados_ECE2009.ppt.

12. Ministerio de Educación del Perú (2005a). Evaluación Nacional del rendimiento estudiantil. Informe pedagógico de resultados. Comprensión de textos escritos: segundo grado de primaria y sexto grado de primaria. Lima: MED.

13. Ministerio de Educación del Perú (2005b). Evaluación Nacional del rendimiento estudiantil. Informe pedagógico de resultados. Comprensión de textos escritos: tercer grado de secundaria y quinto grado de primaria. Lima: MED.

14. Ministerio de Educación del Perú (2004). Una aproximación a la alfabetización lectora de los estudiantes peruanos de 15 años. Resultados del Perú en la evaluación internacional PISA. Lima: MED.

15. Ministerio de Educación del Perú (2003). Cómo rinden los estudiantes peruanos en Comunicación y Matemática: resultados de la Evaluación Nacional 2001. Informe descriptivo. Lima: MED.

16. Siegrist, F. \& Sinclair, H. (1991). Principles of spelling found in the first two grades. En: C. Kamii, M. Manning \& G. Manning (Eds.), Early literacy: a constructivist foundation for whole language. Washington: National Education Association of the United States.

17. Solé, I. (2004). Estrategias de lectura. Barcelona: Editorial Graò.

18. Teberosky, A. (2001). La iniciación en el mundo de lo escrito. En: M. Cerezo et al. (Eds.), Comprensión lectora. El uso de la lengua como procedimiento. Barcelona: Editorial Graò.

19. Tolchinsky, L. \& Pipkin, M. (2001). Seis lectores en busca de un texto. En: M. Cerezo et al. (eds.), Comprensión lectora. El uso de la lengua como procedimiento. Barcelona: Editorial Graò. 